\title{
BLOQUEO ANDROGÉNICO INTERMITENTE (BAI) EN CÁNCER DE PRÓSTATA AVANZADO. ¿POR QUÉ NO EL TRATAMIENTO ESTÁNDAR?
}

\author{
Dominique Prapotnich, Rafael Sánchez-Salas, Xavier Cathelineau, Oleksandr Stakhovskyi, José \\ E.A. Rocha Jr y Guy Vallancien.
}

Departamento de Urología. Institut Mutualiste Montsouris. Universidad Paris Descartes. París. Francia.

Resumen.- OBJETIVO: Revisar la bibliografía y presenter una visión contemporánea del bloqueo androgénico en el cáncer de próstata.

MÉTODOS: Llevamos a cabo una búsqueda bibliográfica en PubMed sobre bloqueo androgénico intermitente (BAll. Para la elaboración del presente artículo utilizamos los artículos obtenidos y la experiencia del Instituto Mutualista Montsouris.

RESULTADOS: El BAl es un abordaje del tratamiento de deprivación androgénica que mantiene un control oncológico eficaz y a la vez evita la morbilidad asociada

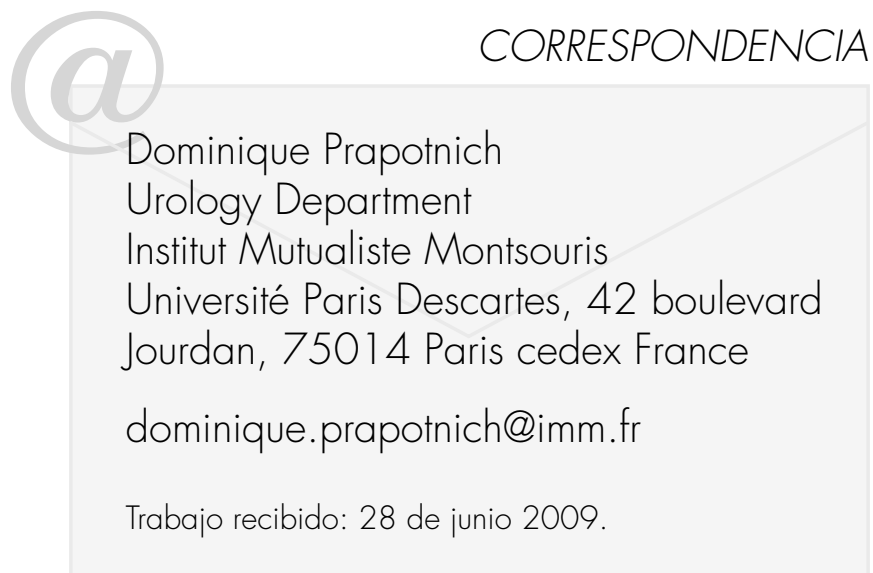

con el bloqueo androgénico continuo. Los matices del BAl han sido evaluados por la comunidad urológica para verificar sus posibles beneficios. Un abordaje basado en la evidencia apoya la idea del BAl como un tratamiento estándar en cáncer de próstata avanzado que necesite bloqueo androgénico. Las variaciones entre los equipos médicos en los criterios de tratamiento y vigilancia están a la espera de estandarización.

CONCLUSIONES: Es necesario revisar el "Gold Standard" del bloqueo androgénico en cáncer de próstata avanzado. La evolución innegable del BAl necesita que la comunidad urológica lo abrace.

Palabras clave: Tratamiento hormonal intermitente. Cáncer de próstata. Bloqueo androgénico. Castración. Antagonistas androgénicos.

Summary.- OBIECTIVES: To review the literature and present a contemporary image of androgen deprivation for prostate cancer.

METHODS: We conducted a PubMed search on intermittent androgen deprivation. Articles obtained on intermittent androgen deprivation (IAD) and the experiences at Institut Montsouris were used for the review.

RESULTS: IAD is an approach to hormonal deprivation that holds effective cancer control while preventing the morbidity associated with continuous androgen blockade. IAD nuances have been assessed by urological community teams in order to verify its possible potential benefits. Evidence based approach supports the idea of IAD as a standard of therapy for advanced prostate cancer requiring hormone deprivation. Variation among medical teams' criteria for the treatment and surveillance await standardization. 
CONCLUSIONS: Reassessing the gold standard of hormonal blockade in advanced prostate cancer is mandatory. The undeniable evolution of IAD needs to be embraced by the urological community.

Keywords: Intermittent hormone treatment. Prostate cancer. Androgen blockade. Castration. Androgen antagonists.

\section{INTRODUCCIÓN}

El Bloqueo Androgénico Intermitente (BAl) ha demostrado de forma extensiva ser factible en manos de varios grupos clínicos alrededor del mundo, aunque las series publicadas son difíciles de evaluar debido a su heterogeneidad en términos de criterios de inclusión. Es interesante que casi todos los estudios han mostrado tres hechos importantes: Primero, que el $\mathrm{BAl}$ es en términos de control oncológico, al menos, igual que el bloqueo androgénico continuo (BAC). Segundo, que hay al menos un beneficio individual para el paciente en términos de recuperación de la testosterona, y tres, que el BAl es menos caro que el BAC por razones obvias. En base a estas tres premisas y nuestra experiencia propia, somos objetivamente entusiastas con el BAl. Aquí, presentamos una revisión concisa, con el objetivo de defender el BAl como un potencial estándar de tratamiento para las mismas indicaciones que ha tenido hasta hoy el BAC.

Los beneficios del bloqueo androgénico para el cáncer de próstata metastásico (BACPM) fueron demostrados hace 68 años por Huggings \& Hodges (1). El BACPM continua siendo un tratamiento paliativo pues la enfermedad eventualmente evoluciona hacia la hormonoresistencia. El BACPM también lleva consigo varios efectos secundarios inmediatos y a largo plazo (Inmediatos: sofocos, fatiga, impotencia sexual, pérdida de libido. Tardíos: Desmineralización osea (3), riesgo de fracturas, pérdida muscular, depresión, aumento de la resistencia a insulina, anemia, malestar, dislipemias, acumulación de grasa en los tejidos. El objetivo del BAl es disminuir los efectos indeseables del BACPM obteniendo a la vez los beneficios del bloqueo androgénico.

La experiencia inicial con bloqueo androgenico intermitente fue publicada por Klotz y cols. en 1986 (2). Esta experiencia pionera estableció el concepto de intervención hormonal a medida y fue llevada a cabo mediante la utilización de estibestrol en la fase de tratamiento (ON) seguida por una fase sin tratamiento (OFF), en función de los valores de PSA y testosterona.
Se han publicado varios estudios experimentales sobre bloqueo androgénico completo. En 1990, Bruchowsky (2) demostró la viabilidad del BAl en tumores de mama en ratones Shionogi, mediante la realización de una fase de tratamiento y una fase de vigilancia sin tratamiento. Este procedimiento permitió un retraso del tiempo a la hormonorresistencia 3 veces superior en comparación con los modelos de tratamiento continuo.

Sato (2) verificó los efectos mencionados del $\mathrm{BAl}$ en su experiencia preliminar con implantes de cáncer de próstata en ganglios linfáticos en un modelo animal.

Estos resultados experimentales apoyaron la utilización de BAI en protocolos clínicos. La idea original era controlar la presión de selección de clones lque en BAC termina llevando a hormonorresistencia), mediante la exposición periódica de las células cancerígenas a andrógenos. Un modelo matemático no lineal desarrolado por Shimada (3) intentaba explicar por qué el BAI puede ser eficaz.

El efecto competitivo juega un papel importante entre las células cancerígenas andrógeno dependientes y las andrógeno-independientes, y se asume que es esencial para el descenso de las células andrógeno-independientes bajo un nivel normal de andrógenos.

Hoy en día varios estudios de fase II (3-4) han probado la viabilidad, toxicidad más baja y buena tolerancia del BAl, que mejora la calidad de vida por la disminución de los efectos secundarios del bloqueo androgénico durante el periodo de vigilancia sin tratamiento.

\section{Meta análisis}

Un meta análisis publicado en 2006 (2) combinaba los resultados de 10 protocolos con más de 50 pacientes disponibles en la literatura. Nuestra institución colaboró con datos de 411 pacientes de los 1446 incorporados en el análisis final de este trabajo. Lo más destacable de este meta análisis era el criterio de heterogenicidad para las fases ON/OFF de tratamiento y sin tratamiento del BAI entre los diferentes grupos en diferentes continentes. La fase libre de tratamiento (OFF) tiende a ser más prolongada en Estados Unidos y más corta en Europa. No se detectaron diferencias en supervivencia en los casos localmente avanzados, con tratamiento menos de 5 meses y sin tratamiento más de 8 meses. Por otro lado, en pacientes con enfermedad metastásica importante, un bloqueo androgénico de más de 8 meses demos- 
tró ser beneficioso. En este análisis 366 pacientes tenían enfermedad metastásica en hueso o ganglios. 1080 pacientes no tenían metástasis, 517 recibieron BAl como tratamiento primario y 563 recibieron un tratamiento inicial primario y se les indicó BAI en el momento de la recidiva bioquímica.

EI PSA inicial y el nadir de PSA fueron claramente identificados como potentes factores de predicción de la supervivencia libre de enfermedad (PSA inicial < $10 \mathrm{ng} / \mathrm{dl}$ mejor que >10; el nadir, cuanto menor, mejor).

El abordaje recomendado para el BAl fue un bloqueo androgénico completo mediante la utilización de un antiandrógeno no esteroideo y un análogo de LH-RH. Se eligió un nivel de PSA de $15 \mathrm{ng} / \mathrm{ml}$ como punto de reinicio del tratamiento buscando obtener la mejor relación entre los periodos de tratamiento y no tratamiento. Un PSA menor de $4 \mathrm{ng} / \mathrm{ml}$ es considerado ampliamente como el umbral para empezar la vigilancia sin tratamiento.

\section{Institut Mutualiste de Montsouris}
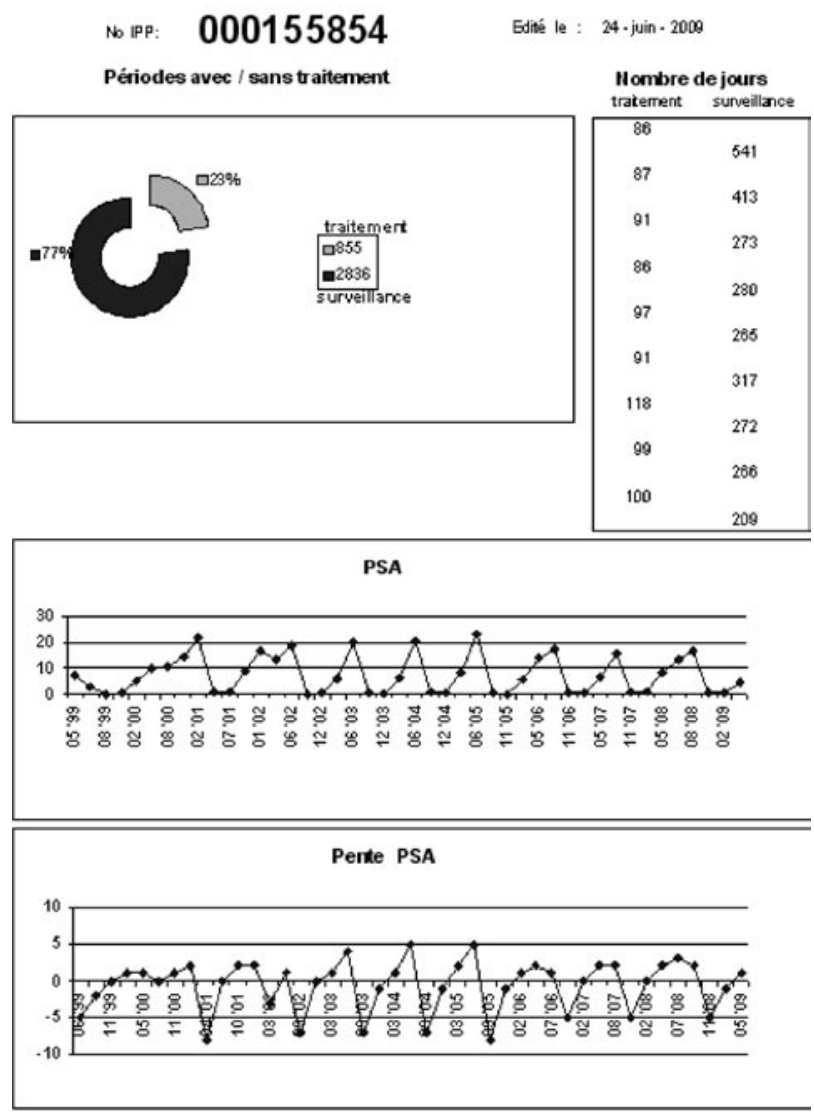

FIGURA 1
Este oportuno trabajo ha demostrado la viabilidad y seguridad del BAl en un grupo grande de pacientes. El BAl se confirmó como una opción lógica de tratamiento para cualquier paciente considerado previamente para bloqueo androgénico continuo.

\section{Experiencia de Montsouris con BAl}

Los resultados y recomendaciones presentados en el meta análisis anteriormente citado reflejan los de nuestra serie en términos de resultados y puntos de corte para tratamiento y vigilancia. Nuestra experiencia temprana comenzó en 1992 y continúa, con 566 pacientes que han seguido tratamiento con $\mathrm{BAl}$ en el IMM. Los resultados iniciales y actualizaciones han sido publicados previamente $(2,3)$ En resumen, nuestros criterios de PSA para las fases de tratamiento y vigilancia son $20 \mathrm{ng} / \mathrm{dl}$ y 4 $\mathrm{ng} / \mathrm{dl}$ respectivamente (Figural). Los pacientes con enfermedad metastásica importante no son buenos candidatos para BAI. De los 566 pacientes tratados, 218 recibieron $\mathrm{BAl}$ después de tratamiento primario y recidiva bioquímica posterior: y 348 pacientes con enfermedad micro o macrometastásica iniciaron BAI sin ningún otro abordaje terapéutico previo. Hemos tratado un grupo de pacientes con una mediana de edad de 74,47 años con una mediana de PSA de $17 \mathrm{ng} / \mathrm{ml}$ y una mediana de seguimiento en nuestra serie de hasta 84 meses. Los pacientes más antiguos en nuestra experiencia han recibido 13 ciclos (Fase

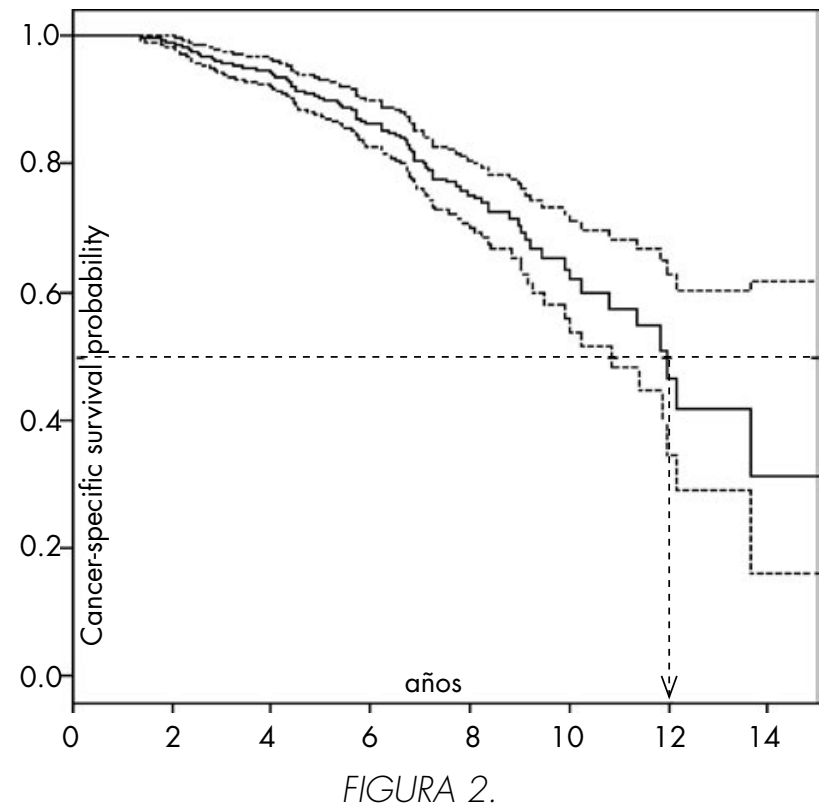


ON de tratamiento+ Fase Off sin tratamiento) y cuando comparamos el ciclo de inducción con el $6^{\circ}$ ciclo observamos una diferencia en la mediana de la duración (23 meses vs 10 meses). La duración del ciclo alcanza una meseta en el 13 er ciclo. El $32 \%$ de los pacientes desarrollaron enfermedad hormonorresistente durante el seguimiento. La mortalidad cáncer específica fue del $13 \%$. En esta serie se verificó una mediana de supervivencia cáncer específica de 12 meses (Figura 2).

EI PSA, la puntuación de Gleason, y la edad inicial fueron identificados como factores predictivos para la supervivencia.

\section{Estudios aleatorizados}

El oportuno estudio de Goldenberg y cols. (2) define los puntos óptimos para el BAl utilizando el nivel del PSA y estableciendo los fundamentos para los deseados ensayos clínicos Fase III.

Se han llevado a cabo varios estudios aleatorizados que comparan BAl y BAC, estando sus resultados disponibles. Salonen y cols. (3) realizaron el Estudio Prostático Finés VII para identificar pacientes que podrían beneficiarse del BAI. Un total de 856 hombres con cáncer de próstata localmente avanzado o metastásico fueron incluidos y recibieron bloqueo androgénico durante 24 semanas. Los respondedores al bloqueo androgénico fueron asignados aleatoriamente, en una proporción uno a uno, a bloqueo androgénico continuo o BAl. Los criterios de inclusión fueron disminución del PSA a menos de 10 $\mathrm{ng} / \mathrm{ml}$ o mayor del $50 \%$ si era menor de $20 \mathrm{ng} / \mathrm{ml}$ al inicio. 564 pacientes (665) fueron asignados a BAl o BAC. El grupo de pacientes que no cumplía criterios de inclusión tenía valores significativamente mayores del PSA, la fosfatasa alcalina, los porcentajes de tumores T4, de tumores pobremente diferenciados, de enfermedad metastática y el número de zonas "calientes" óseas en enfermedad M1. Estos pacientes con enfermedad muy avanzada probablemente no sean buenos candidatos para BAI.

El protocolo más importante en términos metodológicos es el estudio SEUG de Calais da Silva y cols. (3) que utilizaron los mismos criterios racionales que en nuestra institución. Ellos presentaron 766 pacientes con cáncer de próstata localmente avanzado o metastásico que recibieron un tratamiento de inducción de 3 meses. Los pacientes con una disminución del PSA hasta $<4 \mathrm{ng} / \mathrm{ml}$ o del $80 \%$ del valor inicial (626) fueron incluidos en el estudio. En la inducción se utilizaron acetato de ciproterona y hormona liberadora de la hormona luteinizante (LHRH). En el brazo de tratamiento continuo se utilizó bloqueo androgénico completo. No hubo diferencias de supervivencia entre BAl y BAC. Aunque hubo un número mayor de muertes por cáncer en el grupo de BAl; el número de muertes cardiovasculares fue mayor en el brazo de BAC. Los pacientes que recibieron BAl referían una mejor satisfacción sexual. Los autores no verificaron ninguna diferencia entre $B A I$ y $B A C$ en términos de progresión de la enfermedad o supervivencia global después de un periodo de seguimiento de 8 años. Estos resultados van probablemente a favorecer al $\mathrm{BAl}$ en un análisis posterior durante el seguimiento. Por otra parte, los elementos de calidad de vida y especialmente la potencia sexual eran significativamente mejores en el grupo de BAl. En el estudio se recomendó un periodo de tratamiento en fase $\mathrm{ON}$ de tres meses y reinicio cuando el PSA se elevaba por encima de $20 \mathrm{ng} / \mathrm{ml}$

Un artículo de Leval (3) también encuentra una tasa de progresión menor en el brazo de BAI frente a BAC $(7 \%$ vs. $38,9 \%)$. Comunican un ensayo clínico que asignaba aleatoriamente 68 pacientes con cáncer de próstata avanzado a BAl o BA continuo con acetato de goserelina y flutamida. Después de una mediana de seguimiento de 30,8 meses, la tasa de progresión andrógeno independiente fue significativamente menor en el brazo de tratamiento intermitente que en el de continuo $(7 \%$ vs. $38,9 \%)$. Los pacientes que recibían tratamiento intermitente estuvieron sin tratamiento el $59 \%$ del tiempo.

El protocolo aleatorizado de Mottet (3) no encontró ninguna diferencia significativa en supervivencia o calidad de vida comparando BAl y BAC; sin embargo, este estudio incluía pacientes con enfermedad metastásica importante (mediana PSA 558-663) y una supervivencia global corta (entre 3 y 4 años), lo que impide la comparación.

Nuestra experiencia concuerda con la de Salonen y cols. (13) en cuanto a lo inadecuado del BAI en el carcinoma prostático metastasico importante.

Irani y cols. (3) también han publicado sus resultados que no encuentran ninguna diferencia entre los dos abordajes. Los resultados de este estudio se basan en una comparación de 6 meses de tratamiento y 6 meses de vigilancia, sin control individual del PSA. Este abordaje no se beneficia de las ventajas potenciales del BAl, ya que una parte importante de la fase de tratamiento $\mathrm{ON}$ se da en el supuesto brazo de BAI. La recuperación de la testosterona es esencial en el BAl, y más tarde implica una forma de administración del tratamiento claramente dinámica para beneficiarse de las ventajas de una exposición limitada al bloqueo hormonal. Gulley y cols (3) mos- 
traron que una fase de tratamiento preconcebida podría comprometer la recuperación de testosterona.

En su estudio, después de 6 meses de tratamiento con análogos de la hormona liberadora de gonadotropinas $(\mathrm{GnRH})$ en 80 pacientes, los niveles de testosterona y dihidrotestosterona no volvieron a valores normales hasta una mediana de 14,9 y 16,6 semanas. En el análisis multivariante, otros factores asociados con el retraso en la recuperación de la testosterona incluían edad avanzada, testosterona baja antes del tratamiento y la utilización de análogos de GnRH trimestrales frente a mensuales (3). La fase de vigilancia sin tratamiento es la piedra angular del BAI y se ha visto que la utilización de un inhibidor de la ciclooxigenasa 2 durante la fase sin tratamiento del BAl puede mejorar la eficacia y el tiempo sin tratamiento(4). El gran mensaje sostenido por la literatura con alto nivel de evidencia disponible es que el BAl nunca ha mostrado resultados peores cuando se ha comparado con su contrapartida continua (Con mejores resultados en algunos artículos).

\section{Puntos de discusión}

Todos los datos disponibles han motivado que la comunidad urológica dedicara a este tema una sesión plenaria del Congreso de la Asociación Americana de Urología en Chicago en el 2009. La pregunta del panel fue si el estándar de tratamiento debe ser bloqueo continuo o intermitente. Adicionalmente, era tarea de la mesa redonda verificar la justificación, evolución y resultados del BAI. En base a estos elementos, se estableció claramente que el BAl es un "gold estándar" potencial en carcinoma de próstata avanzado considerando los costes y la eficacia, y también se estableció que es un abordaje menos deletéreo con un control del cáncer equivalente (dos ensayos clínicos internacionales definitivos están en marcha intentando contestar la pregunta de si el BAI o BAC deben ser el tratamiento estándar).

Tannock (3) tiene el honor bien merecido de haber cuestionado el BAC frente a un nuevo estándar potencial representado por el BAI. Estamos al principio de un cambio en el tratamiento hormonal del cáncer de próstata y nuestros pacientes deben convertirse en los beneficiarios naturales de esta evolución. Debe decirse que todavía necesitamos refinar y estandarizar los criterios de inclusión y seguimiento del $\mathrm{BAl}$, lo que sigue siendo una ardua tarea debido al hecho de que el tratamiento tiene que hacerse a medida de cada paciente en base a la agresividad particular de la enfermedad, y este es el único camino para obtener mejores resultados.

\section{CONCLUSIÓN}

El BAl debe ser contemplado actualmente como una opción de tratamiento segura y fiable en cáncer de próstata avanzado (excepto pacientes con enfermedad metastásica importante o PSA >100 ng/ $\mathrm{ml}$ ) en base a la evidencia suministrada por varias instituciones.

La supervivencia y la selección precisa de los pacientes siguen siendo temas pendientes de resolver, a desarrollar en un futuro.

\section{BIBLIOGRAFIA y LECTURAS RECOMENDADAS ( ${ }^{*}$ lectura de interés $y^{* *}$ lectura fundamental)}

**1. Huggins C, Hodges CV. Studies on prostatic cancer. The effect of castration, of estrogen and of androgen injection on serum phosphatases in metastatic carcinoma of the prostate. Cancer Res. 1941; 1:293-97.

2. Spry NA, Galvao DA, Davies R, et al. Long-term effects of intermittent androgen suppression on testosterone recovery and bone mineral density: results of a 33-month observational study. BJU Int. 2009; Mar 5.

*3. Klotz LH, Herr HW, Morse MJ, et al. Intermittent endocrine therapy for advanced prostate cancer. Cancer, 1986; 58:2546-50.

**4. Bruchovsky N, Rennie PS, Coldman AJ, et al. Effects of androgen withdrawal on the stem cell composition of the shionogi carcinoma. Cancer Res. 1990; 50:2275-82.

5. Sato N, Gleave ME, Bruchovsky N, et al. Intermittent androgen suppression delays progression to androgen-independant regulation of prostatespecific antigen gene in the $\mathrm{LNCaP}$ prostate tumor model. J. steroid biochem. mol. biol. 1996; 58:139-146.

*6. Shimada T, Aihara K. A nonlinear model with competition between prostate tumor cells and its application to intermittent androgen suppression therapy of prostate cancer. Math Biocsi, 2008; 214(1-2):134-9.

7. Albrecht W, Collette L, Fava C, et al. Intermittent maximal androgen blockade in patients with metastatic prostate cancer: an EORTC feasibility study. Eur. Urol. 2003; 44:505-11.

8. Lane TM, Ansell W, Farrugia D, et al. Long-term outcomes in patients with prostate cancer managed with intermittent androgen suppression. Urol. Int. 2004; 73:117-22.

9. Shaw GL, Wilson P, Cuzick J, et al. International study into the use of intermittent hormone therapy in the treatment of carcinoma of the prostate: 
meta-analysis of 1446 patients. BJU int. 2007; 99:1056-65.

10. Prapotnich D, Fizazi K, Escudier B, Mombet A, Cathala N, Vallancien G. A 10-year clinical experience with intermittent hormonal therapy for prostate cancer. Eur. Urol. 2003; 43(3):233-9.

*11. Prapotnich D, Cathelineau X, Rozet F, Barret E, Mombet A, Cathala N, et al. A 16-year clinical experience with intermittent androgen deprivation for prostate cancer: oncological results. World J. Urol. 2009; Feb 27.

*12. Goldenberg SL, Bruchovsky N, Gleave ME, Sullivan LD, Akakura K. Intermittent androgen suppression in the treatment of prostate cancer: a preliminary report. Urology, 1995; 45(5):839-44.

**13. Salonen AJ, Viitanen J, Lundstedt S, Ala-Opas M, Taari K, Tammela TL. FinnProstate Group. Finnish multicenter study comparing intermittent to continuous androgen deprivation for advanced prostate cancer: interim analysis of prognostic markers affecting initial response to androgen deprivation. J. Urol. 2008; 180(3):915-9.

**14. Calais da Silva FE, Bono AV, Whelan P, et al. Intermittent androgen deprivation for locally advanced and metastatic prostate cancer: results from a randomised phase 3 study of the south European uroncological group. Eur. Urol. 2009; 55:126977.

*15. De Leval J, Boca P, Yousef E, et al. Intermittent versus continuous total androgen blockade in the treatment of patients with advanced hormone-naive prostate cancer: results of a prospective ran- domized multicenter trial. Clin. Prostate Cancer, 2002; 1:163-71.

16. Mottet N, Goussard M, Loudili S, et al. Intermittent versus continuous maximal androgen blockade in metastatic (D2) prostate cancer patients. A randomized trial. Eur. Urol. Suppl. 2009; 8(4): 131.

17. Irani J, Celhay O, Hubert J, et al. Continuous versus six months a year maximal androgen blockade in the management of prostate cancer: a randomised study. Eur. Urol. 2008; 54:382-91.

18. Gulley JL, Figg WD, Steinberg SM, Carter J, Sartor O, Higano CS, et al. A prospective analysis of the time to normalization of serum androgens following 6 months of androgen deprivation therapy in patients on a randomized phase III clinical trial using limited hormonal therapy. J. Urol. 2005; 173(5):1567-71.

19. Pickles T, Agranovich A, Berthelet E, Duncan GG, Keyes M, Kwan W, et al. British Columbia Cancer Agency, Prostate Cohort Outcomes Initiative. Testosterone recovery following prolonged adjuvant androgen ablation for prostate carcinoma. Cancer, 2002; 94(2):362-7.

20. Di Silverio F, Sciarra A, Gentile V. Etoricoxib and intermittent androgen deprivation therapy in patients with biochemical progression after radical prostatectomy. Urology, 2008; 71(5):947-51.

**21. Seruga B, Tannock IF. Intermittent androgen blockade should be regarded as standard therapy in prostate cancer. Nat. Clin. Pract. Oncol. 2008; 5(10):574-6. 